\title{
Transcriptome Analyses Reveal Lipid Metabolic Process in Liver Related to the Difference of Carcass Fat Content in Rainbow Trout (Oncorhynchus mykiss)
}

\author{
Guo Hu, Wei Gu, Peng Sun, Qingli Bai, and Bingqian Wang \\ National and Local Joint Engineering Laboratory for Freshwater Fish Breeding, Heilongjiang River Fishery Research Institute, \\ Chinese Academy of Fishery Sciences, Harbin 150070, China
}

Correspondence should be addressed to Bingqian Wang; bingqianwang@yeah.net

Received 2 March 2016; Revised 11 June 2016; Accepted 13 July 2016

Academic Editor: Mihai Miclăuş

Copyright (C) 2016 Guo Hu et al. This is an open access article distributed under the Creative Commons Attribution License, which permits unrestricted use, distribution, and reproduction in any medium, provided the original work is properly cited.

\begin{abstract}
Excessive accumulation of carcass fat in farm animals, including fish, has a significant impact on meat quality and on the cost of feeding. Similar to farmed animals and humans, the liver can be considered one of the most important organs involved in lipid metabolism in rainbow trout (Oncorhynchus mykiss). RNA-seq based whole transcriptome sequencing was performed to liver tissue of rainbow trout with high and low carcass fat content in this study. In total 1,694 differentially expressed transcripts were identified, including many genes involved in lipid metabolism, such as $L-F A B P$, adiponectin, PPAR- $\alpha, P P A R-\beta$, and IGFBP1a. Evidence presented in this study indicated that lipid metabolic process in liver may be related to the difference of carcass fat content. The relevance of PPAR- $\alpha$ and PPAR- $\beta$ as molecular markers for fat storage in liver should be worthy of further investigation.
\end{abstract}

\section{Introduction}

Excessive fat accumulation in farm animals, including fish, has a significant impact on meat quality and the cost of feeding $[1,2]$. The management of fat deposition in farm animals has drawn increasing attention from a growing number of researchers and the public. As we know, body fatness is a heritable, quantitative trait in rainbow trout (Oncorhynchus mykiss), and genetic selection has been successfully used to change the muscle fat content in rainbow trout $[3,4]$. A good understanding of the physiological mechanisms of fat deposition could be essential to genetic enhancement of the production performance of rainbow trout.

Rainbow trout originated in North America and was introduced to China from the Democratic People's Republic of Korea (North Korea) in 1959 [5, 6]. According to statistical data provided by the Food and Agriculture Organization (FAO), rainbow trout is the major cold-water fish farmed in China, and the production amount was approximately 23,000 tons in 2013 [7]. Additionally, in many research areas, such as immunology and environmental carcinogenesis, this organism has been used as a model animal [8]. The draft genome has been sequenced and several genetic materials and genomics tools have been developed for research [9], facilitating our understanding of the whole genomic physiological mechanisms of the fatness trait in rainbow trout.

Similar to farm animals and humans, lipogenesis, triglyceride accumulation, and metabolic homeostasis occur essentially in the liver of rainbow trout [10]. The liver can be considered one of the most important organs involved in lipid metabolism, and the different expression of functional genes involved in lipid synthesis, degradation, transportation, and storage has been analyzed in the livers of trout strains divergently selected for muscle fat content [11-13]. The research showed that genes involved in lipid metabolism might be influenced by divergent selection and lead to a different model of fat storage in the genetically selected lean and fat trout strains [13]. Another later study indicated that lipid and glucose metabolism was regulated by insulin in two experimental rainbow trout lines [14]. In addition, some evidence has suggested that TOR signaling pathway-associated lipogenesis could be overactivated and the utilization of 
glucose in the liver might be also improved by the genetic selection $[11,15,16]$.

In the present study, RNA-seq based transcriptome sequencing was performed to liver tissue of rainbow trout with extremely high and low carcass fat content. The objectives were to identify the differential expressed transcripts and get meaningful information about the molecular mechanism of fat deposition. The results may be helpful in determining more powerful target genes associated with carcass fat content and would potentially be applied in the development of molecular genetic markers for fatness traits in rainbow trout.

\section{Materials and Methods}

2.1. Experimental Animals and Sampling. In this study, a freshwater strain of rainbow trout, selected for large-size body weight and measurement traits using family based BLUP method, was used as experimental material [17]. The breeding program was performed at the Bohai cold-water fish experimental station, located near Jingbo Lake (latitude $44.02^{\circ} \mathrm{N}$, longitude $128.74^{\circ} \mathrm{E}$ ), in Heilongjiang Province, China. Water was supplied to the tanks and ponds at the Bohai experimental station using natural flowing spring water $\left(5.2-18.0^{\circ} \mathrm{C}\right)$ with a water flow of $20-30 \mathrm{~L} / \mathrm{sec}$ and a dissolved oxygen concentration ranging from 7 to $11 \mathrm{mg} / \mathrm{L}$. Seventy-five full-sibling families were established using 75 male and 75 female fish for each generation based on passive integrated transponder (PIT) tag. After each family was separately hatched from eggs, the fish absorbed their yolk sacs (30 to 35 days) before swimming up and beginning to feed on a commercial trout fry diet. Each candidate family was then reared separately in a tank (1-meter diameter, 0.5meter water depth, average water temperature ranging from approximately $6.5^{\circ} \mathrm{C}$ to $12^{\circ} \mathrm{C}$, and water flow at 6 to $8 \mathrm{~L} / \mathrm{min}$ ) for 10 months until reaching a size of $>50 \mathrm{~g}$. Fifty fish with no deformities were randomly selected from each tank with PIT tags and were then deposited together in cemented pools (5-meter width, 30-meter length, and 0.7-meter water depth) for one year. The fish beyond two years of age were cultured in earth ponds (15-meter width, 120-meter length, and 0.8-meter water depth). It takes approximately 3-3.5 years to reach sexual maturity for rainbow trout at the Bohai station. All of the fish were fed according to the feeding program for trout from BioMar. All of the animal work was conducted according to the guidelines for the care and use of experimental animals established by the Ministry of Science and Technology of China (document number: 2006398) and was approved by the Academic Ethics Committee of Heilongjiang River Fishery Research Institute.

Before the traits were measured, the fish were anaesthetized using $0.5 \mathrm{mg} / \mathrm{L}$ 2-phenoxyethanol $\left(\mathrm{C}_{8} \mathrm{H}_{10} \mathrm{O}_{2}\right)$ to avoid injury from handling. Each fish was measured for carcass fat content using a Fish Fatmeter (Distell Company, Old Levenseat, Scotland, UK) (Model FFM-692; calibration: TROUT-2; representing fat content of whole carcass including belly cavity and fish roe) [18], for body weight using electronic scales $(0.1 \mathrm{~g})$, and for total length using Vernier calipers $(0.1 \mathrm{~cm})$; each fish was sexed using secondary sexual characteristics, and at the same time, body measurement traits were assessed. All of phenotypic records of carcass fat content related to 1,422 individuals at 2.5 years of age were used for the calculation of the population mean (7.14 \pm $1.52 \%)$. Six female fish were used for transcriptome sequencing. To ensure that every individual within and between the high and low carcass fat content groups used for RNA sequencing had the same genetic background but no direct kinship, a coancestry analysis was performed based on PIT tag ID of the individuals and the pedigree records. Three female rainbow trout with low carcass fat content (4.20 \pm $0.31 \%$ ) and the same number of high carcass fat content female ones $(11.27 \pm 0.31 \%)$ were randomly selected from the population for transcriptome sequencing. There were extremely significant differences (approximately 3 -fold) in carcass fat content $(p<0.001)$ but no significant difference in body weight between the two groups $(p>0.05)$.

\subsection{Total RNA Extraction, RNA-Seq Library Construction,} and Sequencing. The livers of three low carcass fat content female fish and three high carcass fat content female fish were sampled under RNAse-free conditions to perform RNA extraction for sequencing. All of the tissue samples were frozen in liquid nitrogen and stored at $-80^{\circ} \mathrm{C}$ until analysis. Total RNA was isolated using Trizol reagent (Invitrogen, Carlsbad, CA, USA), and RNA degradation and contamination were monitored on $1 \%$ agarose gels. Then, the clustering and sequencing were performed by the Experimental Department of Novogene Ltd. The clustering of the indexcoded samples was performed on a cBot Cluster Generation System using a TruSeq SR Cluster Kit v3-cBot-HS (Illumina), according to the manufacturers' instructions. After cluster generation, the library preparations were sequenced on an Illumina HiSeq 2500 platform, and 125 bp paired-end reads were generated.

\subsection{RNA-Seq Data Processing, Annotation, and Differential} Expression Identification. Raw counts of RNA-seq reads for each transcript and in each sample were derived and normalized to fragments per kilobase of exon per million fragments mapped reads (FPKM). All transcriptome data from six individuals were aligned to the Oncorhynchus mykiss mRNA dataset (Oncorhynchus mykiss genome project accession via http://www.genoscope.cns.fr/trout/) using the TopHat [19], and the expression of trout transcripts was evaluated using the software Cufflinks [20]; TopHat and Cufflinks were performed with default parameters. Differentially expressed transcripts (fold changes $\geq 2$ or fold changes $\leq 0.5$ and adjusted $p$ value $\leq 0.01$ ) between high and low carcass fat content fish were identified with the Cufflink package based on FPKM; visualization of transcripts expression was performed using $\mathrm{R}$ software [21]. GO terms were assigned to the transcripts according to their corresponding homologs in the trout mRNA database (http://www.genoscope.cns.fr/trout/). The GO annotation result was visualized in WEGO [22]. Lastly, the GO terms enrichment analysis for the differentially expressed transcripts was carried out by topGO package [23]. 
TABLE 1: The information of the primer pairs used to analyze gene expression by real-time quantitative RT-PCR.

\begin{tabular}{|c|c|c|}
\hline Standard nomenclature ${ }^{*}$ & Primer & Product size (bp) \\
\hline \multirow{2}{*}{ GSONMT00021034001 } & $5^{\prime}$ CTGGGCACCACCTACTCAT $3^{\prime}$ & \multirow{2}{*}{110} \\
\hline & $5^{\prime}$ GTGTCTGTGAAGGCAACG 3' & \\
\hline \multirow{2}{*}{ GSONMT00026574001 } & $5^{\prime}$ AAACTGCTGGTGAAGACG $3^{\prime}$ & \multirow{2}{*}{136} \\
\hline & $5^{\prime}$ TGTTGAGGACAAAGAGGG 3' & \\
\hline \multirow{2}{*}{ GSONMT00028411001 } & $5^{\prime}$ CCCTGGGCGTACAGTTTGAC $3^{\prime}$ & \multirow{2}{*}{188} \\
\hline & $5^{\prime}$ CTTGGCATCCACTCCATCGT 3' & \\
\hline \multirow{2}{*}{ GSONMT00029574001 } & $5^{\prime}$ TTCAAGGGTCGTGGGAGA 3' & \multirow{2}{*}{159} \\
\hline & $5^{\prime}$ CGGAGGGTGTTGGAAGTG $3^{\prime}$ & \\
\hline \multirow{2}{*}{ GSONMT00032762001 } & $5^{\prime}$ AGCAGAACGGCAATGACT $3^{\prime}$ & \multirow{2}{*}{181} \\
\hline & $5^{\prime}$ TCCTGGACGCTGGAGAAT $3^{\prime}$ & \\
\hline \multirow{2}{*}{ GSONMT00051137001 } & $5^{\prime}$ GGAGCCTGGTTGTGGATG $3^{\prime}$ & \multirow{2}{*}{286} \\
\hline & $5^{\prime}$ GCTGTGGCCGTGGAGATA $3^{\prime}$ & \\
\hline \multirow{2}{*}{ GSONMT00051158001 } & $5^{\prime}$ CCTGGCTCTGTTTGTGGC $3^{\prime}$ & \multirow{2}{*}{137} \\
\hline & 5’ TGTCGTCTGGGTGGTTGG 3' & \\
\hline \multirow{2}{*}{ GSONMT00054447001 } & $5^{\prime}$ CTGGAGTAAAGATGGGTGAC $3^{\prime}$ & \multirow{2}{*}{169} \\
\hline & 5’ GTCCTGTTCTGGGATTGG 3’ & \\
\hline \multirow{2}{*}{ GSONMT00060652001 } & 5' CCGACCACCAACCCTAAT 3' & \multirow{2}{*}{272} \\
\hline & $5^{\prime}$ CACTGGCAGCGGTAGAAC $3^{\prime}$ & \\
\hline \multirow{2}{*}{ GSONMT00062349001 } & $5^{\prime}$ GCCTGGATGAGAATGA 3’ & \multirow{2}{*}{335} \\
\hline & $5^{\prime}$ GATACCGCAGGACAAT $3^{\prime}$ & \\
\hline \multirow{2}{*}{ GSONMT00064682001 } & $5^{\prime}$ CCTACTGAGCCCATTCCT $3^{\prime}$ & \multirow{2}{*}{360} \\
\hline & $5^{\prime}$ ATGGAGACTAAGCGAGGC 3’ & \\
\hline \multirow{2}{*}{ GSONMT00070501001 } & $5^{\prime}$ CGCCCACCTCTAAACAAGCC $3^{\prime}$ & \multirow{2}{*}{459} \\
\hline & $5^{\prime}$ GCAGCGTCATCCAGCCCATC 3' & \\
\hline \multirow{2}{*}{ GSONMT00077785001 } & $5^{\prime}$ CGTGCCCATCCGTTTCAATA $3^{\prime}$ & \multirow{2}{*}{137} \\
\hline & $5^{\prime}$ CCCGAGCATCTTTGGTGTAG 3' & \\
\hline \multirow{2}{*}{ GSONMT00082167001 } & $5^{\prime}$ ACCGTGGGAGTAGTTCTTGC 3' & \multirow{2}{*}{425} \\
\hline & $5^{\prime}$ TAGACACCGTTGTAGACCAG $3^{\prime}$ & \\
\hline \multirow{2}{*}{$\mathrm{EF} 1 \alpha$} & $5^{\prime}$ TCCTCTTGGTCGTTTCGCTG 3' & \multirow{2}{*}{159} \\
\hline & $5^{\prime}$ ACCCGAGGGACATCCTGTG $3^{\prime}$ & \\
\hline
\end{tabular}

${ }^{*}$ Note: the standard nomenclature for rainbow trout according to GBrowse-2.54 of rainbow trout genome project database.

2.4. Validation of Differentially Expressed Genes by RealTime Quantitative RT-PCR. Fourteen differentially expressed genes identified by the transcriptome sequencing were validated by real-time quantitative RT-PCR, using the same fish sample, and EF1 $\alpha$ was used as a reference control [11]. Real-time RT-PCR was performed using SYBR (R) GREEN I NUCLEIC A (Life Technologies) on the LightCycler 480II Real-Time System (Roche, Switzerland). The reaction was performed using the following conditions: denaturation at $95^{\circ} \mathrm{C}$ for $3 \mathrm{~min}$, followed by 40 cycles of amplification $\left(95^{\circ} \mathrm{C}\right.$ for $30 \mathrm{~s}, 60^{\circ} \mathrm{C}$ for $30 \mathrm{~s}$, and $60^{\circ} \mathrm{C}$ for $45 \mathrm{~s}$ ). Relative expression was calculated using the delta-delta-Ct method; primer sequences can be found in Table 1.

\section{Results}

3.1. Sequencing of Liver Transcriptome in Rainbow Trout. The liver transcriptome expression of six fish at 2.5 years was analyzed by RNA sequencing. After removing low-quality reads, adaptor, and barcode sequences, nearly 35 million clean reads for each individual were obtained; the descriptive statistics of the RNA-seq reads for each individual are shown in Table 2. All of the raw data were submitted to the NCBI database (accession numbers SRX1067664 and SRR2072562).

3.2. Gene Functional Annotation for the Differentially Expressed Transcripts. A total of 1,694 differentially expressed transcripts were identified from liver tissue between the high and low carcass fat content rainbow trout (Figure 1). The standard nomenclature, expression, and gene annotation for the differentially expressed transcripts were shown in Table S1 in Supplementary Material available online at http://dx.doi.org/10.1155/2016/7281585. Among these transcripts, 912 transcripts were highly expressed in high carcass fat content fish, including many key factors of lipogenesis and fat deposition in rainbow trout, such as peroxisome proliferator activated receptor alpha $(P P A R-\alpha), P P A R-\beta$, insulin-like growth factor binding protein la (IGFBP1a), 
Color key

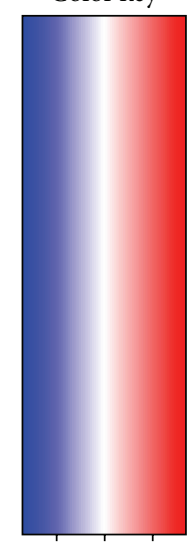

$\begin{array}{llll}-5 & 0 & 5\end{array}$

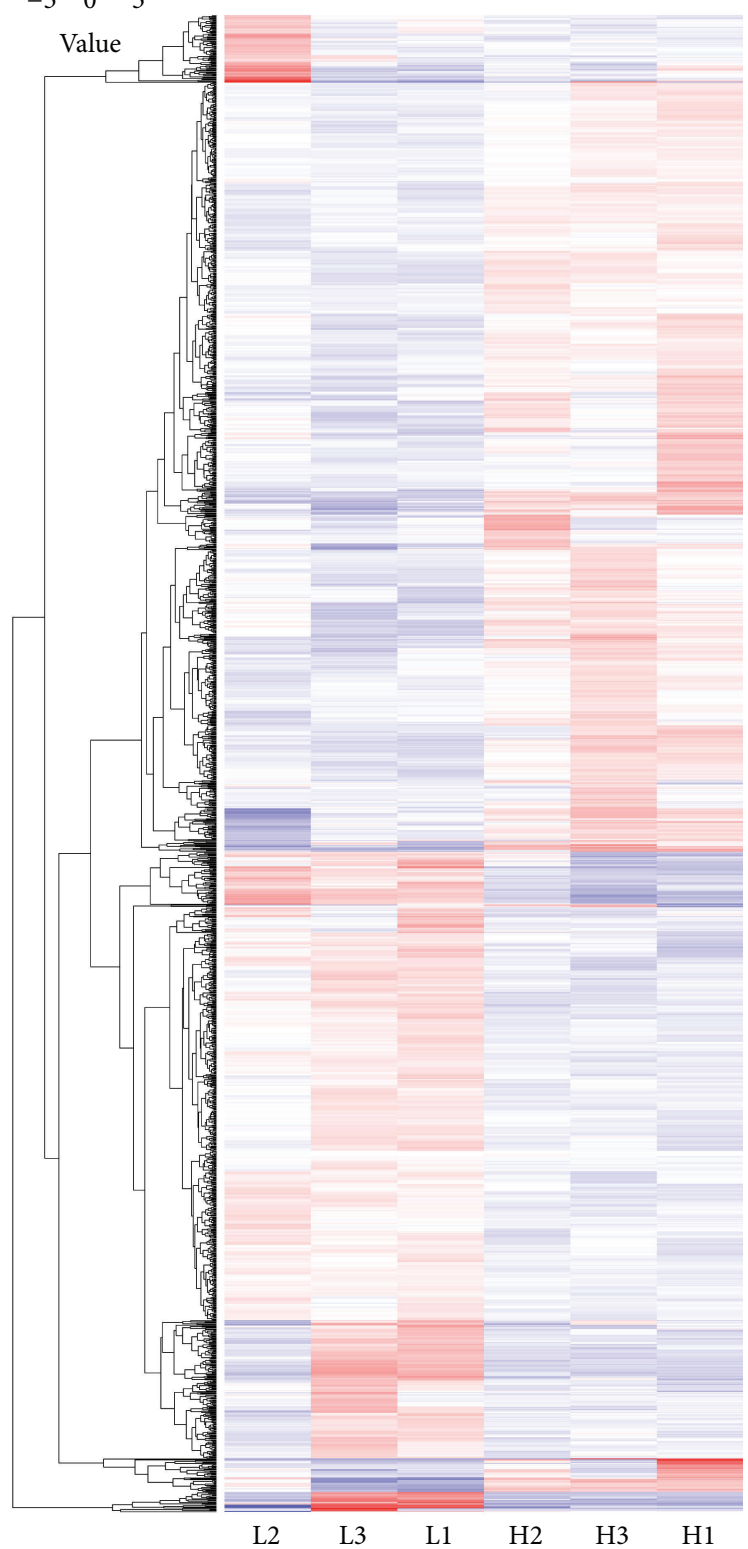

FIGURE 1: Cluster image of 1,694 significant differentially expressed transcripts between the high and low carcass fat content rainbow trout. A total of 1,694 differentially expressed transcripts were identified with Cufflink package based on FPKM: 912 were highly expressed in rainbow trout with high carcass fat content, and 782 showed higher expression level in those fish with low carcass fat content. H1, H2, and H3 represent the first, second, and third fish in the high carcass fat content group, respectively; and L1, L2, and L3 represent the first, second, and third fish in the low carcass fat content group, respectively. Colored bars indicate relative expression levels. Transcripts expressed at higher levels were assigned red, while transcripts expressed at low levels were assigned blue. 
TABLE 2: The descriptive statistics of the raw RNA-seq datasets for all of the six individuals sequenced in the present study.

\begin{tabular}{|c|c|c|c|c|c|c|c|}
\hline Sample & Raw reads & Clean reads & Clean bases & Error (\%) & Q20 (\%) & Q30 (\%) & GC (\%) \\
\hline H1_1 & 17429887 & 16907974 & $2.11 \mathrm{G}$ & 0.03 & 96.27 & 92.45 & 50.25 \\
\hline H1_2 & 17429887 & 16907974 & $2.11 \mathrm{G}$ & 0.04 & 93.31 & 87.75 & 50.2 \\
\hline $\mathrm{H} 2 \_1$ & 18051923 & 17574994 & $2.2 \mathrm{G}$ & 0.03 & 96.18 & 92.3 & 50.37 \\
\hline $\mathrm{H} 2 \_2$ & 18051923 & 17574994 & $2.2 \mathrm{G}$ & 0.04 & 93.56 & 88.15 & 50.33 \\
\hline H3_1 & 16777106 & 16241788 & $2.03 \mathrm{G}$ & 0.03 & 96.16 & 92.23 & 50.07 \\
\hline H3_2 & 16777106 & 16241788 & $2.03 \mathrm{G}$ & 0.04 & 93.33 & 87.71 & 50.04 \\
\hline L1_1 & 18535584 & 17957775 & $2.24 \mathrm{G}$ & 0.03 & 96.41 & 92.72 & 49.7 \\
\hline $\mathrm{L} 1 \_2$ & 18535584 & 17957775 & $2.24 \mathrm{G}$ & 0.04 & 93.97 & 88.84 & 49.66 \\
\hline L2_1 & 18083733 & 17537741 & $2.19 \mathrm{G}$ & 0.03 & 96.26 & 92.43 & 49.72 \\
\hline L2_2 & 18083733 & 17537741 & $2.19 \mathrm{G}$ & 0.04 & 94.03 & 88.89 & 49.68 \\
\hline L3_1 & 19420929 & 18772655 & $2.35 \mathrm{G}$ & 0.03 & 96.24 & 92.41 & 50.08 \\
\hline L3_2 & 19420929 & 18772655 & $2.35 \mathrm{G}$ & 0.04 & 93.66 & 88.3 & 50.05 \\
\hline
\end{tabular}

Notes: sample name: $\mathrm{H} 1$ is the first fish in high carcass fat content group and so on; L1 is the first fish in high carcass fat content group and so on; H1_1 is the left-end reads, H1_2 is the right-end reads; the descriptive statistics for the individual H1 is the sum of H1_1 and H1_2 and so on.

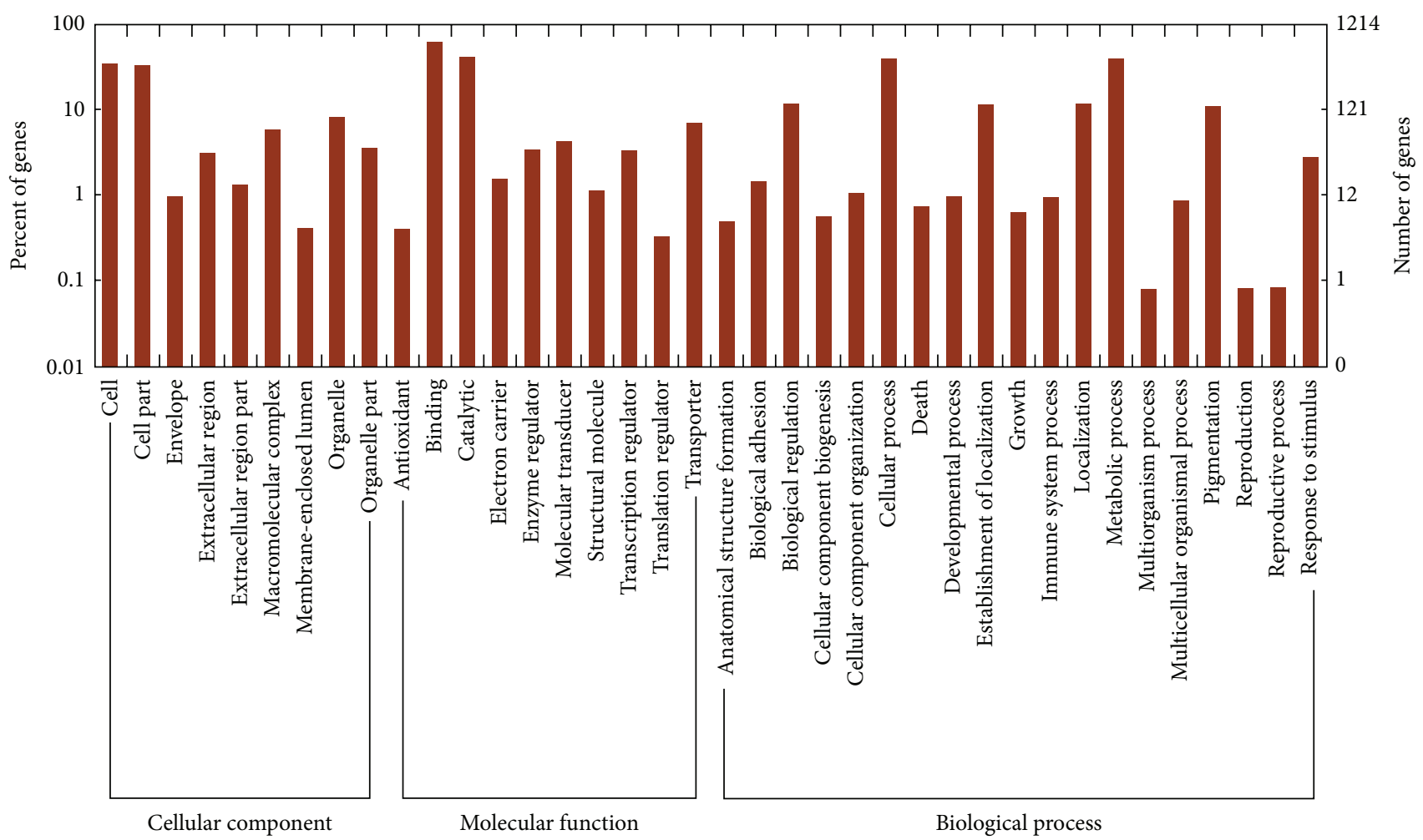

FIGURE 2: GO annotation results of the differently expressed transcripts between the high and low carcass fat content rainbow trout.

and fatty acid-binding protein $10-\mathrm{A}$, liver $(L-F A B P)$. At the same time, 782 transcripts showed higher expression levels in low carcass fat content fish, including many transcription factors with important effects on growth, differentiation, lipid metabolism, carbohydrate metabolism, and immunity, for example, growth hormone receptor isoform 1 (GHR1), insulin-induced gene 1 (INSIG1), and heat shock $70 \mathrm{kDa}$ protein (HSP70).

GO annotations provided in the trout mRNA database (Oncorhynchus mykiss genome project accession via http://www.genoscope.cns.fr/trout/) were used in this study. At least one in three main categories of GO terms (biological process, molecular function, and cellular component) was assigned to all 1,694 differentially expressed transcripts and then was classified into different functional categories, as shown in Figure 2. The results showed that metabolic process (GO: 0008152) was the most abundant group in the biological process category, followed by cellular process (GO: 0009987), while biological regulation (GO: 0065007) and establishment of localization (GO: 0051234) were also common, consistent 
with the central role of liver in the basal and intermediary metabolism. In the molecular function category, the most abundant group was binding (GO: 0005488), and the second most abundant one was catalytic activity (GO: 0003824). There were also transcripts classified into specific groups, such as transporter activity (GO: 0005215), molecular transducer activity (GO: 0060089), enzyme regulator activity (GO: 0030234), and transcription regulator activity (GO: 0030528), indicating that many genes were involved in important physiological functions of synthesis, transport, and catabolism in the livers of rainbow trout. In the cellular component category, the most abundant groups were related to cell (GO: 0005623), cell part (GO: 0044464), organelle (GO: 0043226), organelle part (GO: 0044422), and macromolecular complex (GO: 0032991).

3.3. GO Enrichment Analysis for Differentially Expressed Transcripts. The results of GO enrichment analysis for 1,694 differentially expressed transcripts were shown in Table S2 in the Supplementary Material. GO terms in the biological process category were highly enriched, with $84 \mathrm{GO}$ terms attaining significant levels, including GO: 0044710 (single-organism metabolic process), GO: 0055114 (oxidation-reduction process), GO: 0006520 (cellular amino acid metabolic process), GO: 0006629 (lipid metabolic process), GO: 0006006 (glucose metabolic process), GO: 0019318 (hexose metabolic process), GO: 0005996 (monosaccharide metabolic process), and GO: 0006541 (glutamine metabolic process), consistent with the physiological functions of the livers of rainbow trout. At the same time, the GO terms in the molecular function category were also highly enriched, and $88 \mathrm{GO}$ terms reached the significant level, including GO: 0016491 (oxidoreductase activity), GO: 0016298 (lipase activity), GO: 0048037 (cofactor binding), GO: 0050662 (coenzyme binding), GO: 0005319 (lipid transporter activity), and GO: 0004465 (lipoprotein lipase activity), indicating that the lipid metabolism mode might be different between high and low carcass fat content fish.

Moreover, we analyzed the expression of transcripts grouped as "lipid metabolic process" GO term (0006629) based on the transcriptome data, revealing that 52 of 372 transcripts (14\%) were differentially expressed between the high and low carcass fat content fish groups (Figure 3), providing meaningful information about the difference in lipid metabolism between the two groups. The standard nomenclature, expression, and gene annotation for the 52 transcripts were shown in Table S3.

3.4. Validation of Differentially Expressed Genes by Real-Time Quantitative RT-PCR. To validate the results of differentially expressed genes in transcriptome sequencing, we performed real-time quantitative RT-PCR for 14 differentially expressed genes involved in lipid metabolism, growth regulation, and other important functions. Among these genes, $L-F A B P$, putative I-FABP, IGFBP1a, insulin-like growth factor binding protein, acid labile subunit (IGFBP-ALS), adiponectin, cytochrome P450, PPAR- $\alpha, P P A R-\beta$, and lipopolysaccharidebinding protein $(L B P)$ were highly expressed in the high carcass fat content fish group, while heat shock $70 \mathrm{kDa}$ protein (HSP70), acetyl-CoA acetyltransferase, cytosolic (ACAT2), StAR-related lipid transfer protein (SATRT), GHR1, and INSIG1 showed lower expression level in the high carcass fat content fish group. The results of differentially expressed genes identified by real-time quantitative RT-PCR had good consistency with the transcriptome data. For transcriptome data, the evaluation criteria for differentially expressed genes between the high and low carcass fat content fish groups were fold changes $\geq 2$ or fold changes $\leq 0.5$ and adjusted $p$ value $\leq 0.01$, while for real-time quantitative RT-PCR we used Student's $t$-test $(p<0.05)$. All fourteen genes detected by real-time RT-PCR reached significance. The fold change results of RNA sequencing and real-time RT-PCR for 14 differentially expressed genes were compared in Table 3.

\section{Discussion}

As is well known, the Chinese believe that the best tasting and most nutritious aquatic animals must be alive immediately prior to cooking, and they eat whole fish from beginning to end (with head and tail); thus, the fat content of the whole carcass of the fish body is an economically important trait in China. Fatty acid de novo synthesis in rainbow trout occurs mainly in the liver [10,24], and several studies have explored the correlations between gene expression in liver tissues and lipid metabolism [25-27].

4.1. Differentially Expressed Functional Genes Involved in Lipid Metabolism and Energy Balance. Among the 1,694 differentially expressed genes, $L-F A B P$ was detected to be more than 30 -fold higher expressed in the fat group than in the lean group by both transcriptome sequencing and real-time RT-PCR methods. Liver type fatty acid-binding protein is a functional protein with a small molecular weight that belongs to a superfamily of lipid-binding proteins and participates in intracellular fatty acid transportation in human, farm animals, birds, and fish [28-30]. Higher expression of the $L$ $F A B P$ gene in the livers of the high carcass fat content group than in the low carcass fat content group might be the result of increased fatty acid transportation into white muscle, skin, and other parts, leading to more fat deposition in the whole carcass. The results indicated that the expression of the $L$ $F A B P$ gene should have a positive effect on fat deposition in the rainbow trout.

The PPAR gene superfamily belongs to the nuclear receptor superfamily, including three different genes-PPAR$\alpha, P P A R-\beta$, and PPAR- $\gamma$. PPARs are a type of ligand-activated transcription factors that control gene expression by binding to specific response elements (PPREs) within promoters [31]. PPARs play critical physiological roles as lipid sensors and regulators of lipid metabolism and can transactivate multiple target genes and interact with other transcription factors involved in lipid metabolic pathways [32,33]. In the current study, PPAR- $\alpha$ and PPAR- $\beta$ were expressed significantly more in the high carcass fat content fish group than in the low carcass fat content fish group by transcriptome sequencing and as validated by real-time RT-PCR. Our results regarding 


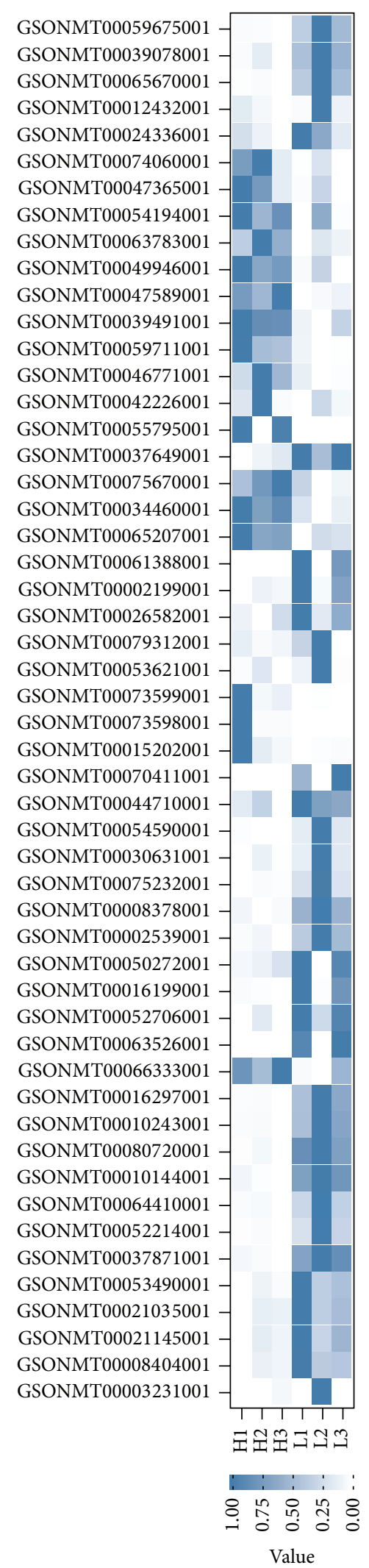

Figure 3: Heat map showing the expression profiles of differentially significant transcripts involved in lipid metabolic processes. A total of 52 of 372 transcripts involved in lipid metabolic processes (GO: 0006629) were differentially expressed between the high and low carcass fat content fish groups. H1, H2, and H3 represent the first, second, and third fish in the high carcass fat content group, respectively; L1, L2, and L3 represent the first, second, and third fish in the low carcass fat content group, respectively. Colored bars indicate relative expression levels. Transcripts expressed at higher levels were assigned blue, while transcripts expressed at low levels were assigned white. 
TABLE 3: Comparison of fold differences in 14 differentially expressed genes detected by transcriptome sequencing and real-time RT-PCR analyses.

\begin{tabular}{lccl}
\hline Standard nomenclature & $\begin{array}{c}\text { Fold change by real-time } \\
\text { RT-PCR }\end{array}$ & Fold change by RNA-seq & Gene annotation \\
\hline GSONMT00021034001 & $0.25 \pm 0.03$ & 0.18 & Heat shock 70 kDa protein \\
GSONMT00026574001 & $1.40 \pm 0.12$ & 2.70 & $\begin{array}{l}\text { Lipopolysaccharide-binding protein/bactericidal } \\
\text { permeability-increasing protein }\end{array}$ \\
GSONMT00028411001 & $4.84 \pm 0.35$ & 4.44 & Fatty acid-binding protein, intestinal putative mRNA \\
GSONMT00029574001 & $36.83 \pm 2.30$ & 17.70 & Cytochrome P450 \\
GSONMT00032762001 & $33.50 \pm 1.95$ & 35.73 & Fatty acid-binding protein 10-A, liver \\
GSONMT00051137001 & $6.05 \pm 0.50$ & 4.27 & Insulin-like growth factor binding protein la \\
GSONMT00051158001 & $1.50 \pm 0.15$ & 3.03 & Peroxisome proliferator activated receptor alpha \\
GSONMT00054447001 & $0.18 \pm 0.04$ & 0.18 & Acetyl-CoA acetyltransferase, cytosolic \\
GSONMT00060652001 & $2.22 \pm 0.15$ & 2.66 & Peroxisome proliferator activated receptor beta \\
GSONMT00062349001 & $0.13 \pm 0.02$ & 0.19 & StAR-related lipid transfer protein \\
GSONMT00064682001 & $2.80 \pm 0.15$ & 2.76 & Insulin-like growth factor binding protein, acid labile \\
GSONMT00070501001 & $0.26 \pm 0.03$ & 0.11 & subunit \\
GSONMT00077785001 & $3.22 \pm 0.19$ & 17.14 & Growth hormone receptor isoform 1 \\
GSONMT00082167001 & $0.71 \pm 0.05$ & 0.29 & Adiponectin \\
\hline
\end{tabular}

* Note: the standard nomenclature for rainbow trout according to GBrowse-2.54 of rainbow trout genome project database.

PPAR- $\alpha$ expression were different from Kolditz et al's results on the divergent selection of lean and fat fish for muscle fat content by cDNA microarray in rainbow trout [13]. In their work, PPAR- $\alpha$ expression was lower in the fat line than in the lean line. The differences between our results and the result of Kolditz et al. might have been caused in part by different ages of the fish and varying aquaculture environmental conditions. Other potential reasons for the difference in PPAR- $\alpha$ expression between the high and low fat content fish in the two populations included the different histories of the two populations. In addition, in contrast to PPAR- $\alpha$ and $P P A R-\beta$, the expression of PPAR- $\gamma$ in the liver was very low and was not differentially expressed between the fat and lean group, consistent with the expression level of $P P A R-\gamma$ gene in liver tissue being only $10 \%$ to $30 \%$ of the level in adipose tissue in mammals $[34,35]$ and similar to that in the chicken [36]. In the current study, the gene expression of $P P A R-\alpha$ and $P P A R-\beta$ was highly correlated with fat deposition, suggesting that they were also key factors of fat accumulation in the rainbow trout carcass.

Cytochrome P-450 is transcriptionally modulated by glucocorticoid binding to glucocorticoid response elements in the promoter region, playing important roles in the oxidative metabolism of endogenous and exogenous compounds [37]. Cytochrome P-450 was detected to be more than 30 -fold higher expressed in the fat group than in the lean group by transcriptome sequencing, and it was validated to be nearly 20 -fold higher by real-time RT-PCR. This result was in agreement with the currently known oxidation-reduction process of gene expression in the liver. The insulin regulated lipid metabolism of fish does not seem to follow mammalian patterns; the insulin-like growth factors (IGFs) are polypeptides with high sequence similarity to insulin, and they are able to modulate lipolysis and lipogenesis in fish hepatocytes [14-16, 38]. Insulin-like growth factor binding proteins (IGFBPs) regulate the biological functions of IGFs, and IGFs can stimulate the growth of multiple tissue cell types [39]. In the current study, the gene expression of two IGFBP isoforms and gene expression of INSIG1 were differently expressed between the lean and fat fish groups, suggesting potential roles of insulin and insulin-like growth factors in lipid storage or utilization of fat accumulation in rainbow trout.

In total, many functional genes involved in lipid and carbohydrate metabolism might contribute to phenotypic variations in carcass fat content, and they were found to be expressed in both high and low carcass fat content fish groups. However, some of these genes were expressed significantly different between these two groups. On the one hand, this phenomenon could partly occur because of potential positive genetic correlation between growth traits and carcass fat content and the growth traits being under strong artificial selection in this experimental population. On the other hand, there was a large difference in the group mean of carcass fat content between the lean and fat groups, suggesting that the dynamics of genetics effects and the gene expression mode might be influenced by genetic heterogeneity between the lean and fat groups.

4.2. Metabolic Process Related to the Difference of Carcass Fat Content Based on GO Enrichment Analysis. The GO approach could help to predict the functions of genes based on the existing architecture and prior knowledge of molecular biological mechanisms for nonmodel organisms. In the present study, a large number of functional genes were significantly 
enriched in lipid and carbohydrate metabolism related GO terms, such as GO: 0006629 (lipid metabolic process), GO: 0006006 (glucose metabolic process), GO: 0019318 (hexose metabolic process), and GO: 0005996 (monosaccharide metabolic process), which was consistent with liver being an important tissue in the biological process of lipid and carbohydrate metabolism and metabolic energy balance. In detail, we identified 52 differentially expressed transcripts significantly enriched in the lipid metabolic process (GO: 0006629) and 20 differentially expressed transcripts significantly enriched in the glucose metabolic process (GO: 0006006). In farm animals, fatty acids and glucose are the two main metabolic substrates oxidized by animals for energy production. The mobilization of fat stores could be decreased by glucose, using both inhibiting lipolysis and stimulating primary reesterification approaches. Our results were consistent with this information and also suggested that the expression and regulation of lipid and carbohydrate metabolism genes should play key roles in the ontogenesis of carcass fat content in rainbow trout.

\subsection{Consistency between Transcriptome Sequencing and Real-} Time RT-PCR. As is known, transcriptome sequencing and real-time RT-PCR analysis are both powerful tools for obtaining a view of gene expression. However, quantifying gene expression by sequencing and real-time RT-PCR is not necessarily consistent. Although much meaningful information can be obtained from transcriptome sequencing alone, this technology still has limitations in accuracy and in fairness for RNA-seq data processing [40]. Although fold changes were different for some genes between these two methods, all fourteen of the genes detected by real-time RT-PCR attained significant levels of differential expression $(p<0.05)$, conferring robustness to our transcriptome dataset.

\section{Conclusion}

In this study, the transcript expression profile of the liver tissue in rainbow trout was investigated comprehensively using RNA sequencing and was confirmed by real-time RTPCR. A total of 1,694 transcripts were differentially expressed between high and low carcass content fish group. Evidence presented in this study indicated that lipid metabolic process in liver may be related to the difference of carcass fat content. The relevance of PPAR- $\alpha$ and PPAR- $\beta$ as molecular markers for fat storage in liver commands further investigation.

\section{Competing Interests}

The authors declare that they have no competing interests regarding the publication of this paper.

\section{Acknowledgments}

This study was supported by the Special Fund for Agro-Scientific Research in the Public Interest (201403012), National Natural Science Foundation of China (31502157), and the
Central-Level Non-Profit Scientific Research Institutes Special Funds (HSY201501). The authors acknowledge Dr. Youyi Kuang and Dr. Xianhu Zheng for their technical support in the phenotypic data collection of the carcass fat content.

\section{References}

[1] J. D. Wood, M. Enser, A. V. Fisher et al., "Fat deposition, fatty acid composition and meat quality: a review," Meat Science, vol. 78, no. 4, pp. 343-358, 2008.

[2] D. Tobin, A. Kause, E. A. Mäntysaari et al., "Fat or lean? The quantitative genetic basis for selection strategies of muscle and body composition traits in breeding schemes of rainbow trout (Oncorhynchus mykiss)," Aquaculture, vol. 261, no. 2, pp. 510521, 2006.

[3] C. Weil, A.-S. Goupil, E. Quillet, L. Labbe, and F. Le Gac, “Twoway selection for muscle lipid content modifies puberty and gametogenesis in rainbow trout," Cybium, vol. 32, no. 2, p. 198, 2008.

[4] E. Quillet, S. Le Guillou, J. Aubin, and B. Fauconneau, "Twoway selection for muscle lipid content in pan-size rainbow trout (Oncorhynchus mykiss)," Aquaculture, vol. 245, no. 1-4, pp. 49$61,2005$.

[5] D. Sun and B. Wang, "Aquaculture of salmonids in China," Chinese Journal Fisheries, vol. 23, no. 2, pp. 56-62, 2010.

[6] Z. Wang and Y. Yang, "Cold water fish culture in China," in Cold Water Fisheries in the Trans-Himalayan Countries, T. Petr and D. B. Swar, Eds., vol. 431, FAO, Fisheries Technical Paper, Rome, Italy, 2002.

[7] FAO, "Aquaculture production: quantities 1950-2013," FISHSTAT Plus.

[8] G. H. Thorgaard, G. S. Bailey, D. Williams et al., "Status and opportunities for genomics research with rainbow trout," Comparative Biochemistry and Physiology $-B$ Biochemistry and Molecular Biology, vol. 133, no. 4, pp. 609-646, 2002.

[9] C. Berthelot, F. Brunet, D. Chalopin et al., "The rainbow trout genome provides novel insights into evolution after wholegenome duplication in vertebrates," Nature Communications, vol. 5, article 3657, 2014.

[10] R. J. Henderson and J. R. Sargent, "Lipid biosynthesis in rainbow trout, Salmo gairdnerii, fed diets of differing lipid content," Comparative Biochemistry and Physiology Part C: Comparative Pharmacology, vol. 69, no. 1, pp. 31-37, 1981.

[11] S. Skiba-Cassy, M. Lansard, S. Panserat, and F. Médale, "Rainbow trout genetically selected for greater muscle fat content display increased activation of liver TOR signaling and lipogenic gene expression," American Journal of Physiology-Regulatory Integrative and Comparative Physiology, vol. 297, no. 5, pp. R1421-R1429, 2009.

[12] J. Jin, F. Médale, B. S. Kamalam, P. Aguirre, V. Véron, and S. Panserat, "Comparison of glucose and lipid metabolic gene expressions between fat and lean lines of rainbow trout after a glucose load," PLoS ONE, vol. 9, no. 8, Article ID e105548, 2014.

[13] C.-I. Kolditz, G. Paboeuf, M. Borthaire et al., "Changes induced by dietary energy intake and divergent selection for muscle fat content in rainbow trout (Oncorhynchus mykiss), assessed by transcriptome and proteome analysis of the liver," $B M C$ Genomics, vol. 9, article 506, 2008.

[14] J. Jin, S. Panserat, B. S. Kamalam, P. Aguirre, V. Véron, and F. Médale, "Insulin regulates lipid and glucose metabolism similarly in two lines of rainbow trout divergently selected for 
muscle fat content," General and Comparative Endocrinology, vol. 204, pp. 49-59, 2014.

[15] W. Dai, S. Panserat, J. A. Mennigen et al., "Post-prandial regulation of hepatic glucokinase and lipogenesis requires the activation of TORC1 signalling in rainbow trout (Oncorhynchus mykiss)," Journal of Experimental Biology, vol. 216, no. 23, pp. 4483-4492, 2013.

[16] M. Lansard, S. Panserat, E. Plagnes-Juan, I. Seiliez, and S. Skiba-Cassy, "Integration of insulin and amino acid signals that regulate hepatic metabolism-related gene expression in rainbow trout: role of TOR," Amino Acids, vol. 39, no. 3, pp. 801-810, 2010.

[17] G. Hu, W. Gu, Z. Jiang, Q. Bai, and B. Wang, "Heritability estimates and genetic correlations among body weight and measurement traits of large-size rainbow trout, Oncorhynchus mykiss, in China," Journal of the World Aquaculture Society, vol. 45, no. 6, pp. 691-698, 2014.

[18] Distell.com: Distell fish fatmeter, User and technical manual, version 2.8., 2011.

[19] C. Trapnell, L. Pachter, and S. L. Salzberg, “TopHat: Discovering splice junctions with RNA-Seq," Bioinformatics, vol. 25, no. 9, pp. 1105-1111, 2009.

[20] C. Trapnell, A. Roberts, L. Goff et al., "Differential gene and transcript expression analysis of RNA-seq experiments with TopHat and Cufflinks," Nature Protocols, vol. 7, no. 3, pp. 562578, 2012.

[21] R Core Team, R: a Language and Environment for Statistical Computing, R Foundation for Statistical Computing, Vienna, Austria, 2014.

[22] J. Ye, L. Fang, H. Zheng et al., "WEGO: a web tool for plotting GO annotations," Nucleic Acids Research, vol. 34, supplement 2, pp. W293-W297, 2006.

[23] A. Alexa, J. Rahnenführer, and T. Lengauer, "Improved scoring of functional groups from gene expression data by decorrelating GO graph structure," Bioinformatics, vol. 22, no. 13, pp. 16001607, 2006.

[24] D. J. Cowley and M. A. Sheridan, "Insulin stimulates hepatic lipogenesis in rainbow trout, Oncorhynchus mykiss," Fish Physiology and Biochemistry, vol. 11, no. 1-6, pp. 421-428, 1993.

[25] B. S. Kamalam, F. Médale, L. Larroquet, G. Corraze, and S. Panserat, "Metabolism and fatty acid profile in fat and lean rainbow trout lines fed with vegetable oil: effect of carbohydrates," PLoS ONE, vol. 8, no. 10, Article ID e76570, 2013.

[26] J. Sánchez-Gurmaches, L. Cruz-Garcia, J. Gutiérrez, and I. Navarro, "mRNA expression of fatty acid transporters in rainbow trout: in vivo and in vitro regulation by insulin, fasting and inflammation and infection mediators," Comparative Biochemistry and Physiology Part A: Molecular \& Integrative Physiology, vol. 163, no. 2, pp. 177-188, 2012.

[27] J. S. Ings, M. R. Servos, and M. M. Vijayan, "Hepatic transcriptomics and protein expression in rainbow trout exposed to municipal wastewater effluent," Environmental Science \& Technology, vol. 45, no. 6, pp. 2368-2376, 2011.

[28] R. L. Smathers and D. R. Petersen, "The human fatty acidbinding protein family: evolutionary divergences and functions," Human Genomics, vol. 5, no. 3, pp. 170-191, 2011.

[29] A. Chmurzyńska, "The multigene family of fatty acid-binding proteins (FABPs): function, structure and polymorphism," Journal of Applied Genetics, vol. 47, no. 1, pp. 39-48, 2006.

[30] R. L. Londraville, "Intracellular fatty acid-binding proteins: putting lower vertebrates in perspective," Brazilian Journal of Medical and Biological Research, vol. 29, no. 6, pp. 707-720, 1996.
[31] Y. Guan, Y. Zhang, and M. D. Breyer, "The role of PPARs in the transcriptional control of cellular processes," Drug News and Perspectives, vol. 15, no. 3, pp. 147-154, 2002.

[32] M. Ahmadian, J. M. Suh, N. Hah et al., "PPAR $\gamma$ signaling and metabolism: the good, the bad and the future," Nature Medicine, vol. 99, no. 5, pp. 557-566, 2013.

[33] J. Berger and D. E. Moller, "The mechanisms of action of PPARs," Annual Review of Medicine, vol. 53, pp. 409-435, 2002.

[34] J. M. Peters, I. Rusyn, M. L. Rose, F. J. Gonzalez, and R. G. Thurman, "Peroxisome proliferator-activated receptor $\alpha$ is restricted to hepatic parenchymal cells, not Kupffer cells: implications for the mechanism of action of peroxisome proliferators in hepatocarcinogenesis," Carcinogenesis, vol. 21, no. 4, pp. 823826, 2000.

[35] P. Tontonoz, E. Hu, R. A. Graves, A. I. Budavari, and B. M. Spiegelman, "mPPAR $\gamma 2$ : tissue-specific regulator of an adipocyte enhancer," Genes and Development, vol. 8, no. 10, pp. 1224-1234, 1994.

[36] H. B. Wang, Q. G. Wang, X. Y. Zhang et al., "Microarray analysis of genes differentially expressed in the liver of lean and fat chickens," Animal, vol. 4, no. 4, pp. 513-522, 2010.

[37] C. A. Hasemann, R. G. Kurumbail, S. S. Boddupalli, J. A. Peterson, and J. Deisenhofer, "Structure and function of cytochromes P450:a comparative analysis of three crystal structures," Structure, vol. 3, no. 1, pp. 41-62, 1995.

[38] E. Plagnes-Juan, M. Lansard, I. Seiliez et al., "Insulin regulates the expression of several metabolism-related genes in the liver and primary hepatocytes of rainbow trout (Oncorhynchus mykiss)," Journal of Experimental Biology, vol. 211, no. 15, pp. 2510-2518, 2008.

[39] D. R. Clemmons, "Role of insulin-like growth factor binding proteins in controlling IGF actions," Molecular and Cellular Endocrinology, vol. 140, no. 1-2, pp. 19-24, 1998.

[40] Z. Wang, M. Gerstein, and M. Snyder, "RNA-Seq: a revolutionary tool for transcriptomics," Nature Reviews Genetics, vol. 10, no. 1, pp. 57-63, 2009. 

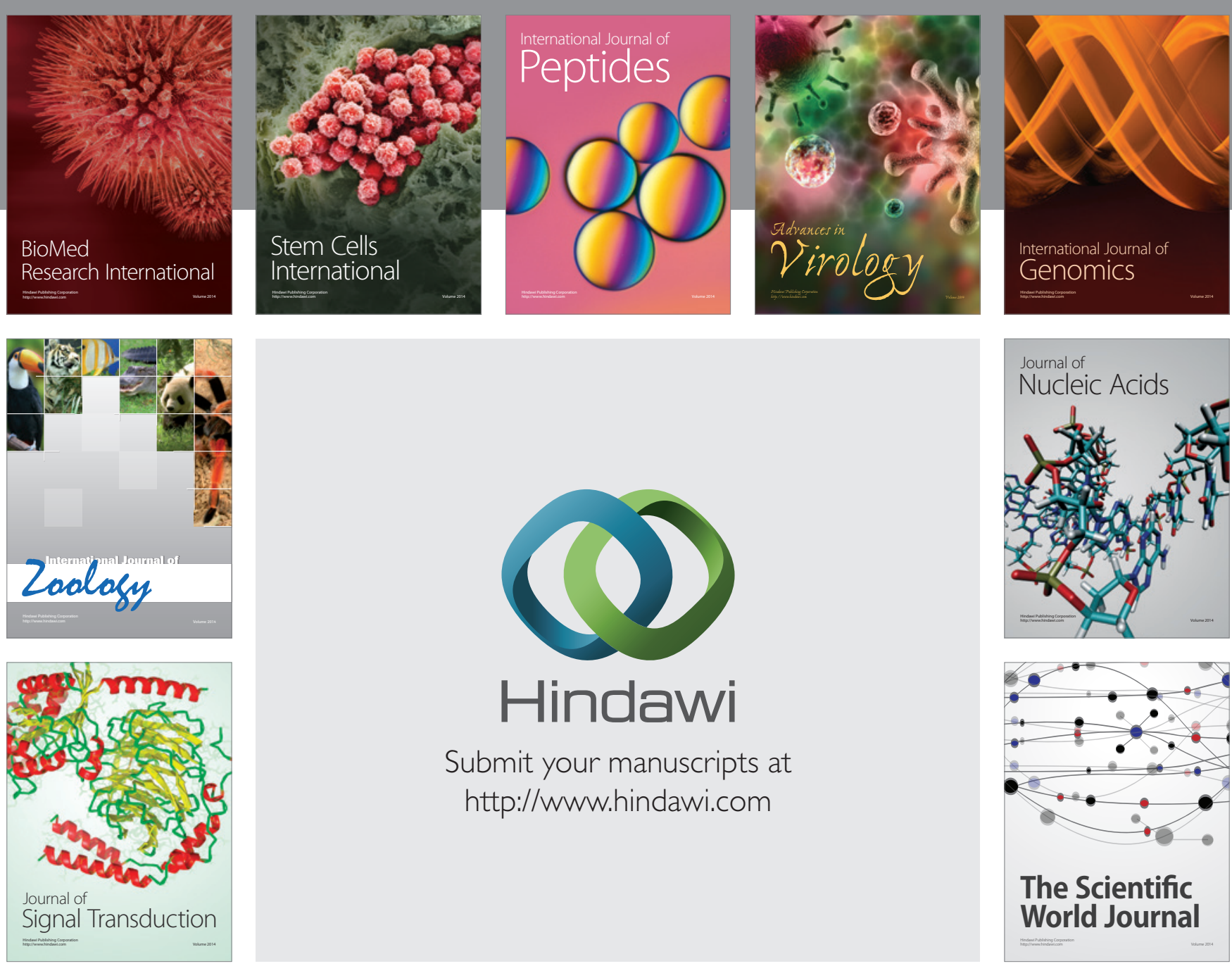

Submit your manuscripts at

http://www.hindawi.com
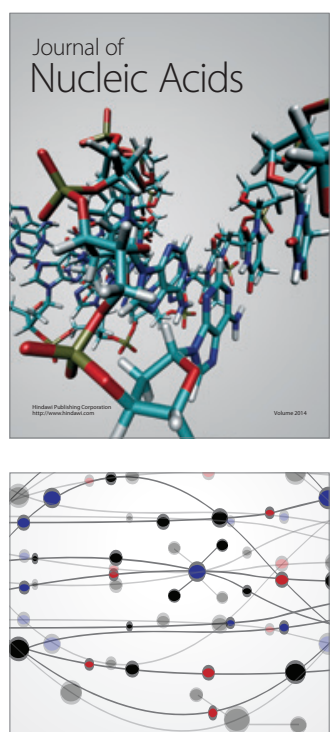

The Scientific World Journal
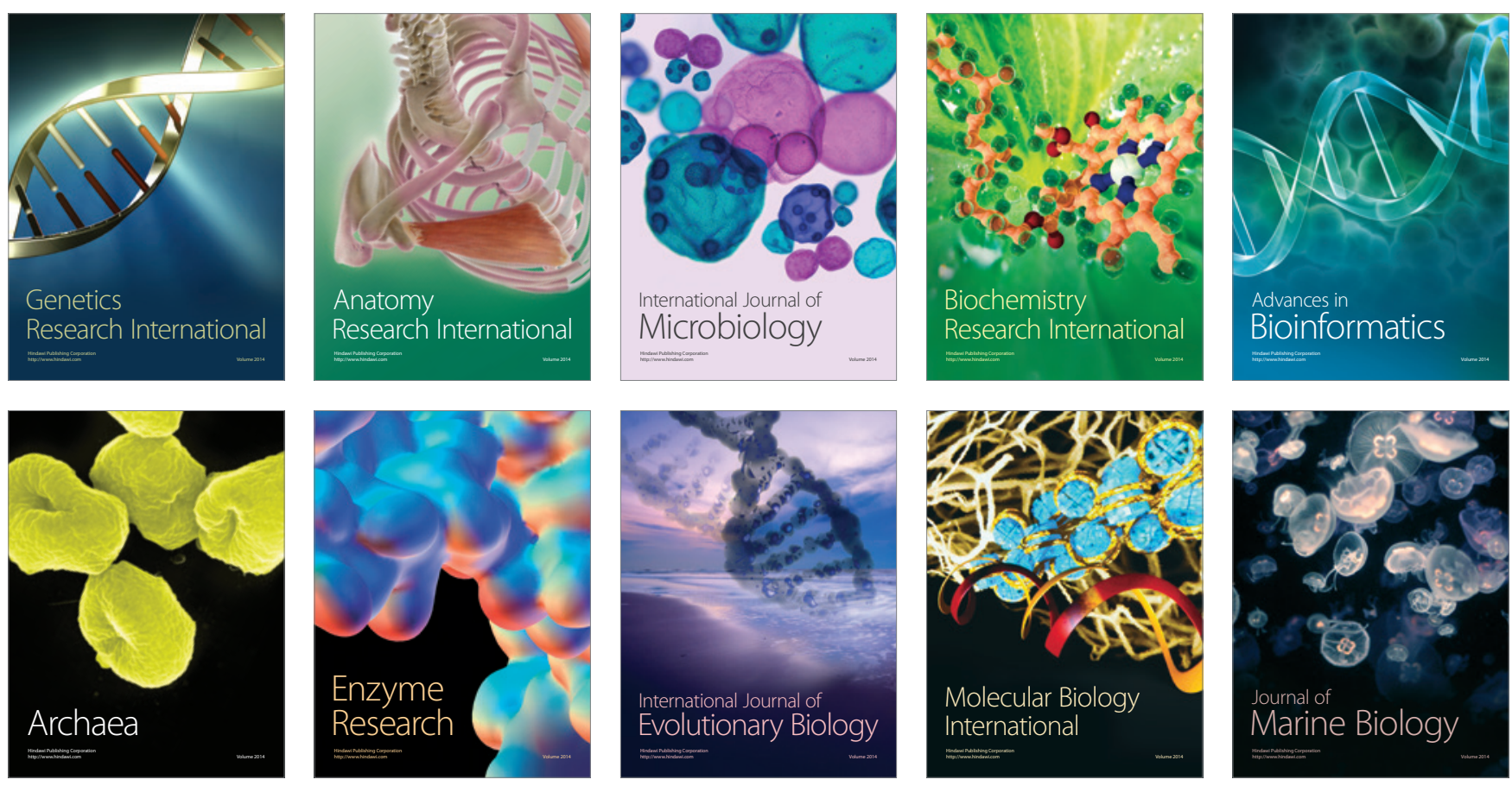